\title{
DOI: https://doi.org/10.24297/jam.v19i.8902
}

\section{Approximation of New Sequence of Integral Type Operators with two Parameters}

\author{
Ali J. Mohammad ${ }^{7}, \quad$ Amal K. Hassan ${ }^{2}$ \\ ${ }^{1}$ Department of Mathematics, College of Education for Pure Science, University of Basrah, \\ Basrah, IRAQ. \\ ${ }^{2}$ Department of Mathematics, College Science, University of Basrah, Basrah, IRAQ. \\ alijasmoh@gmail.com, amalkheleel2015@gmail.com
}

\begin{abstract}
In our paper, we provide and study a new sequence of positive and linear operators of integral type $M_{n, r, s}(f ; y)$. This sequence depends on two parameters, positive integers $r$ and $s$. We mention some of the properties of this sequence and describe a Voronovskaja type asymptotic formula. Besides, we find the error estimates of this approximation in terms of the modulus of continuity. lastly, we introduce a numerical example and compare the results obtained.
\end{abstract}

Keywords: positive and linear operators, Voronovskaja-type asymptotic formula, Modulus of continuity.

Mathematics Subject Classification 2010: 41A10, 41A25, 41A36.

\section{Introduction}

Szasz in 1950, [10] introduced a sequence of positive and linear operators to approximate the unbounded continuous functions in the interval $[0, \infty)$ as:

$Z_{n}(f ; y)=\sum_{l=0}^{\infty} q_{n, k}(y) f\left(\frac{l}{n}\right)$,

where $q_{n, k}(y)=\frac{(n y)^{l}}{l ! e^{n y}}, y \in[0, \infty)$.

After that, several researchers are modified for many sequences of operators [2], [3], and [4].

Rempulska and et.al. in 2009,[9] studied the following sequence of improvement Szasz -Mirakyan operators $Z_{n, r}(f ; y)$ as:

$Z_{n, r}(f(\tau) ; y)=\frac{1}{A_{r}(n y)} \sum_{l=0}^{\infty} \frac{(n y)^{r l}}{(r l) !} f\left(\frac{r l}{n}\right)$,

$y \in[0, \infty), n \in N=\{1,2, \ldots\}$, and for every fixed $r \in N$,

where, $A_{r}(y)=\sum_{l=0}^{\infty} \frac{y^{r l}}{(r l) !}$

clearly $A_{1}(y)=e^{y}$ and $A_{2}(y)=\cosh (y)$.

After that, many researchers presented various studies in this aspect as [1 ], [7 ], [ 8], and [11 ]

Mohammad and Hassan in 2019 [6] introduce a new sequence of integral type operators on the space

$C_{\alpha}[0, \infty)=\left\{f \in C[0, \infty):|f(\tau)|=O\left(e^{\alpha \tau}\right)\right.$, for some $\left.\alpha>0\right\}$

and the norm $\|f\|_{C_{\alpha}[0, \infty)}=\sup _{\tau \in[0, \infty)}|f(\tau)| e^{-\alpha \tau}$ as:

$M_{n, r}(f(\tau) ; y)=\frac{1}{A_{r}(n y)} \int_{0}^{y} A_{r}^{\prime}(n \tau) f(\tau) d \tau$

In the percent paper, we generalized the sequence (1.3) on the space $C_{\alpha}[0, \infty)$ as: 
$M_{n, r, s}(f(\tau) ; y)=\frac{1}{A_{r}(n y)} \int_{0}^{y} A_{r}^{\prime}(n \tau) f\left(y+(\tau-y)^{s}\right) d \tau$

where $r, s \in N$ are parameters. We denote, $A_{r, m}(y)=\sum_{l=0}^{\infty} \frac{y^{r l+m}}{(r l+m) !}, m \in N^{0}$.

Such that, $M_{n, r, s}(f(\tau) ; y)=M_{n, r}(f(\tau) ; y)$ at $\mathrm{s}=1$.

We study the properties of the sequence $M_{n, r, s}(f ; y)$, also, we discuss a formula of Voronovskaja and estimate the error in view of the modulus of continuity. A numerical example for the sequence $M_{n, r, s}(f ; y)$ is given for the test function $f(\tau)=\sin (10 \tau)$ where $\tau \in[0,2]$, Finally, we discuss the results.

\section{Auxiliary Results}

Here, we introduce some properties for the sequence $M_{n, r, s}(f ; y)$

\section{Lemma 2.1}

Let $n, r \in N$ and $y \in[0, \infty)$ we get:

1. $\lim _{n \rightarrow \infty} \frac{A_{r, i}(n y)}{A_{r}(n y)}=1$, for $i \in N$

2. $\lim _{n \rightarrow \infty} \frac{n^{i}}{A_{r}(n y)}=0, i \in N^{0}=\{0,1,2, \ldots\}$

\section{Lemma 2.2}

Let $y \in[0, \infty)$ we have:

(i) $\quad M_{n, r, s}(1 ; y)=1-\frac{1}{A_{r}(n y)} \rightarrow 1$ as $n \rightarrow \infty$;

(ii) $\quad M_{n, r, s}(\tau ; y)=y\left(1-\frac{1}{A_{r}(n y)}\right)-\frac{(-y)^{s}}{A_{r}(n y)}+\frac{(-1)^{s} s !}{n^{s}} \frac{A_{r, s}(n y)}{A_{r}(n y)} \rightarrow y$ as $n \rightarrow \infty$;

(iii) $\quad M_{n, r, s}\left(\tau^{2} ; y\right)=y^{2}\left(1-\frac{1}{A_{r}(n y)}\right)-\frac{(-1)^{s} 2 y}{A_{r}(n y)}+\left\{y^{s}-\frac{s !}{n^{s}} A_{r, s}(n y)\right\}-\frac{1}{A_{r}(n y)}\left\{y^{2 s}-\right.$ $\left.\frac{(2 s) !}{n^{2 s}} A_{r, 2 s}(n y)\right\} \rightarrow y^{2}$ as $n \rightarrow \infty$.

\section{Proof}

We can easily prove this lemma by direct computation.

\section{Definition 2.1}

For $k \in N^{0}$ the $k$ - th order moment $T_{n, k, r}^{s}(y)$ for the operator $M_{n, r, s}(f(\tau) ; y)$ is define as:

$T_{n, k, r}^{s}(y)=M_{n, r, s}\left((\tau-y)^{k} ; y\right)=\frac{1}{A_{r}(n y)} \int_{0}^{y} A_{r}^{\prime}(n \tau)(\tau-y)^{k s} d \tau$

\section{Lemma 2.3}

For the moment function $T_{n, k, r}^{s}(y)$, we obtain:

$$
\begin{aligned}
& T_{n, 0, r}^{s}(y)=1-\frac{1}{A_{r}(n y)} ; \\
& T_{n, 1, r}^{s}(y)=\frac{(-1)^{s}}{A_{r}(n y)}\left\{\frac{s !}{n^{s}} A_{r, s}(n y)-y^{s}\right\} ; \\
& T_{n, 2, r}^{s}(y)=\frac{1}{A_{r}(n y)}\left\{\frac{(2 s) !}{n^{2 s}} A_{r, 2 s}(n y)-y^{2 s}\right\} ; \\
& T_{n, k, r}^{s}(y)=\frac{(-1)^{k s}}{A_{r}(n y)}\left\{\frac{(k s) !}{n^{k s}} A_{r, k s}(n y)-y^{k s}\right\}, k \geq 1 .
\end{aligned}
$$

Further, we have:

(i) $\quad T_{n, k, r}^{s}(y)$ is a polynomial in $y$ not exceed of $k s$, whenever $n$ is sufficiently large.

(ii) for every $y \in[0, \infty), T_{n, k, r}^{s}(y)=O\left(n^{-k s}\right)$. 


\section{Proof}

By using Lemma 2.2 we can prove this lemma immediately.

\section{Lemma 2.4}

For each real number $\alpha, \delta>0$ and $[a, b] \subset(0, \infty)$

$\sup _{y \in[a, b]}\left|\int_{y-t \geq \delta} \frac{A_{r}^{\prime}(n \tau)}{A_{r}(n y)} e^{\alpha \tau} d \tau\right|=O\left(n^{-\lambda}\right), \lambda>0$

\section{Proof}

We can prove this lemma by using Taylor's expansion and (2) with $k=i$ in lemma 2.3,

\section{Main Results}

\section{Theorem 3.1}

For $f \in C_{\alpha}[0, \infty)$, the sequence of positive and linear operators $M_{n, r}(f(\tau) ; y)$ is converge uniformly to $f$ as $n \rightarrow$ $\infty$.

\section{Proof}

From lemmas 2.1 and 2.2 we have:

$$
\begin{aligned}
& M_{n, r, s}(1 ; y) \rightarrow 1 \quad \text { uniformly as } n \rightarrow \infty \\
& M_{n, r, s}(\tau ; y) \rightarrow y \quad \text { uniformly as } n \rightarrow \infty \\
& M_{n, r, s}\left(\tau^{2} ; y\right) \rightarrow y^{2} \quad \text { uniformly as } n \rightarrow \infty
\end{aligned}
$$

Hence, from Korovkin theorem [5], we obtaine:

$\lim _{n \rightarrow \infty} M_{n, r, s}(f(\tau) ; y)=f(y)$ uniformly as $n \rightarrow \infty$

\section{Theorem 3.2}

For $f \in C_{\alpha}[0, \infty), \alpha>0$ and $f$ has two derivatives at a point $y \in(0, \infty)$, we have,

$\lim _{n \rightarrow \infty} n^{s}\left(M_{n, r, s}(f(\tau) ; y)-f(y)\right)=(-1)^{s} s ! f^{\prime}(y)$

\section{Proof}

Applying Taylor's formula for the function $f$, we obtain:

$f(\tau)=f(y)+(\tau-y) f^{\prime}(y)+\frac{(\tau-y)^{2}}{2} f^{\prime \prime}(y)+\xi(\tau, y)(\tau-y)^{2}$, where $\xi(\tau, y) \rightarrow 0$ as $\tau \rightarrow y$.

Operating by $M_{n, r, s}$ we have:

$M_{n, r, s}(f(\tau) ; y)=M_{n, r, s}(1 ; y) f(y)+M_{n, r, s}((\tau-y) ; y) f^{\prime}(y)$

$+\frac{1}{2} M_{n, r, s}\left((\tau-y)^{2} ; y\right) f^{\prime \prime}(y)+M_{n, r, s}\left(\xi(\tau, y)(\tau-y)^{2} ; y\right)$

$M_{n, r, s}(f(\tau) ; y)=T_{n, 0, r}^{s}(y) f(y)+T_{n, 1, r}^{s}(y) f^{\prime}(y)+\frac{1}{2} T_{n, 2, r}^{s}(y) f^{\prime \prime}(y)+R(n, y)$

Where, $R(n, y)=M_{n, r, s}\left(\xi(t, y)(\tau-y)^{2} ; y\right)$,

from Lemmas 2.1 and 2.3 we get

$\lim _{n \rightarrow \infty} n^{s}\left(M_{n, r, s}(f(\tau) ; y)-f(y)\right)=(-1)^{s} s ! f^{\prime}(y)+\lim _{n \rightarrow \infty} n^{s} R(n, y)$.

Now, we prove that $\lim _{n \rightarrow \infty} n^{s} R(n, y) \rightarrow 0$ as $n \rightarrow \infty$.

since $\xi\left(y+(\tau-y)^{s}, y\right) \rightarrow 0$ as $\tau \rightarrow y$, for a given $\varepsilon>0, \exists \delta>0$ such that $\left|\xi\left(y+(\tau-y)^{s}, y\right)\right|<\varepsilon$, whenever 
$0<\left|(\tau-y)^{s}\right|<\delta$, for $\left|(\tau-y)^{s}\right| \geq \delta, \exists C>0$ such that $\left|\xi\left(y+(\tau-y)^{s}, y\right)\right|(\tau-y)^{2 s} \leq C e^{\alpha\left(y+(\tau-y)^{s}\right)}$. Hence,

$n^{s}|R(n, y)| \leq \frac{n^{s}}{A_{r}(n y)} \int_{y-t<\delta} A_{r}^{\prime}(n \tau)\left|\xi\left(y+(\tau-y)^{s}, y\right)\right|(\tau-y)^{2 s} d \tau$

$$
+\frac{n^{s}}{A_{r}(n y)} \int_{y-\tau \geq \delta} A_{r}^{\prime}(n \tau)\left|\xi\left(y+(\tau-y)^{s}, y\right)\right|(\tau-y)^{2 s} d \tau
$$

$:=I_{1}+I_{2}$

$I_{1} \leq n^{s} \varepsilon T_{n, 2, r}^{s}(y)$

$=n^{s} \varepsilon O\left(n^{-2 s}\right)$.

$=\varepsilon O\left(n^{-s}\right)$.

$I_{2} \leq \frac{n^{s}}{A_{r}(n y)} \int_{y-t \geq \delta} A_{r}^{\prime}(n \tau) C e^{\alpha\left(y+(\tau-y)^{s}\right)} d \tau$

Then, from Lemma 2.4, we have:

$I_{2}=C n^{s} O\left(n^{-\lambda}\right)$

$=\operatorname{CO}\left(n^{s-\lambda}\right)$

$=o(1), \lambda>s$

Since $\varepsilon>0$ is arbitrary, so we get $I_{1} \rightarrow 0$ as $n \rightarrow \infty$. As well $I_{2} \rightarrow 0$ as $n \rightarrow \infty$.

Then, $\lim _{n \rightarrow \infty} n^{s} R(n, y) \rightarrow 0$ as $n \rightarrow \infty$.

Now, we find an error for $f \in C_{\alpha}[0, \infty), f \in C_{\alpha}^{1}[0, \infty)$ and $f \in C_{\alpha}^{2}[0, \infty)$.

\section{Theorem 3.3}

If $f \in C_{\alpha}[0, \infty), \alpha>0$ and $x \in[0, \infty)$, then:

$\left|M_{n, r, s}(f(\tau) ; y)-f(y)\right| \leq\left(2+\frac{1}{A_{r}(n y)}\right) \omega_{f}\left(\mu_{s}\right)+\frac{|f(y)|}{A_{r}(n y)}$

Where, $\mu_{s}=M_{n, r, s}(y-\tau ; y)=\frac{(-1)^{s}}{A_{r}(n y)}\left\{y^{s}-\frac{s !}{n^{s}} A_{r, s}(n y)\right\}$

\section{Proof}

For any continuous function $f$, the modulus of continuity is defined as follows:

$\omega_{f}(\delta)=\omega(f, \delta)=\max _{|\tau-y| \leq \delta}|f(\tau)-f(y)|$

since $\omega_{f}(\delta) \geq|f(\tau)-f(y)|$ whenever $|\tau-y| \leq \delta$,

and $f(\tau)-f(y) \leq|f(\tau)-f(y)|$

then, $f(\tau)-f(y) \leq \omega_{f}(|\tau-y|)$

hence, $f(\tau)-f(y) \leq\left(1+\frac{|\tau-y|}{\delta}\right) \omega_{f}(\delta)$

$M_{n, r, s}(f(\tau) ; y) \leq\left(M_{n, r, s}(1 ; y)+\frac{1}{\delta} M_{n, r, s}(y-\tau ; y)\right) \omega_{f}(\delta)+f(x) M_{n, r, s}(1 ; y)$

From Lemma 2.2 we obtain:

$M_{n, r, s}(f(\tau) ; y)-f(y) \leq\left(1-\frac{1}{A_{r}(n y)}+\frac{\mu_{s}}{\delta}\right) \omega_{f}(\delta)-\frac{f(y)}{A_{r}(n y)}$,

$\left|M_{n, r, s}(f(\tau) ; y)-f(y)\right| \leq\left(1+\frac{1}{A_{r}(n y)}+\frac{\mu_{s}}{\delta}\right) \omega_{f}(\delta)+\frac{|f(y)|}{A_{r}(n y)}$ 
Let $\delta=\mu_{s}$, hence

$\left|M_{n, r, s}(f(\tau) ; y)-f(y)\right| \leq\left(2+\frac{1}{A_{r}(n y)}\right) \omega_{f}\left(\mu_{s}\right)+\frac{|f(y)|}{A_{r}(n y)}$

\section{Theorem 3.4}

Suppose that $f \in C_{\alpha}^{1}[0, \infty), \alpha>0$ and $y \in[0, \infty)$, then:

$\left|M_{n, r, s}(f(\tau) ; y)-f(y)\right| \leq\left(\frac{1}{A_{r}(n y)}\right)|f(y)|+\mu_{s}\left|f^{\prime}(y)\right|+\left(\mu_{s}+1\right) \omega_{f^{\prime}}(\delta)$

Where, $\mu_{s}=M_{n, r, s}(y-\tau ; y)=\frac{(-1)^{s}}{A_{r}(n y)}\left\{y^{s}-\frac{s !}{n^{s}} A_{r, s}(n y)\right\}$.

\section{Proof}

By Taylors expansion for the function $f(\tau)$ about $\tau=y$, we obtain:

$f(\tau)-f(y)=(\tau-y) f^{\prime}(y)+(\tau-y)\left(f^{\prime}(\xi)-f^{\prime}(y)\right)$, where $\xi \in(\tau, y)$

Hence,

$$
\begin{aligned}
& f(\tau)-f(y) \leq|\tau-y| f^{\prime}(y)+|\tau-y| \omega_{f^{\prime}}(|\tau-y|) \\
& \leq|\tau-y| f^{\prime}(y)+|\tau-y|\left(1+\frac{|\tau-y|}{\delta}\right) \omega_{f^{\prime}}(\delta) \\
& \begin{aligned}
M_{n, r, s}(f(\tau) ; y)- & M_{n, r, s}(1 ; y) f(y) \\
& \leq M_{n, r, s}((y-\tau) ; y) f^{\prime}(y) \\
& +\left\{M_{n, r, s}((y-\tau) ; y)+\frac{1}{\delta} M_{n, r, s}\left((\tau-y)^{2} ; y\right)\right\} \omega_{f^{\prime}}(\delta)
\end{aligned}
\end{aligned}
$$

Consequently,

$M_{n, r, s}(f(\tau) ; y)-f(y) \leq \frac{1}{A_{r}(n y)} f(y)+\mu_{s} f^{\prime}(y)+\left(\mu_{s}+\frac{\gamma_{s}}{\delta}\right) \omega_{f^{\prime}}(\delta)$

Where $\gamma_{s}=M_{n, r, s}\left((\tau-y)^{2} ; y\right)=\frac{1}{A_{r}(n y)}\left\{\frac{(2 s) !}{n^{2 s}} A_{r, 2 s}(n y)-y^{2 s}\right\}$,

if we take $\delta=\gamma_{s}$ then,

$M_{n, r, s}(f(\tau) ; y)-f(y) \leq \frac{1}{A_{r}(n y)} f(y)+\mu_{s} f^{\prime}(y)+\left(\mu_{s}+1\right) \omega_{f} \cdot\left(\gamma_{s}\right)$

then,

$\left|M_{n, r, s}(f(\tau) ; y)-f(y)\right| \leq \frac{1}{A_{r}(n y)}|f(y)|+\mu_{s}\left|f^{\prime}(y)\right|+\left(\mu_{s}+1\right) \omega_{f^{\prime}}\left(\gamma_{s}\right)$

\section{Theorem 3.5}

Assume that $f \in C_{\alpha}^{2}[0, \infty), \alpha>0$ then for any $n \in N$ it follows that:

$\left|M_{n, r, s}(f(\tau) ; y)-f(y)\right| \leq \frac{1}{A_{r}(n y)}\|f(y)\|+\mu_{s}\left\|f^{\prime}(y)\right\|+\frac{\gamma_{s}}{2}\left\|f^{\prime \prime}(y)\right\|$,

Where, $\mu_{s}=M_{n, r, s}(y-\tau ; y)=\frac{(-1)^{s}}{A_{r}(n y)}\left\{y^{s}-\frac{s !}{n^{s}} A_{r, s}(n y)\right\}$ and $\gamma_{s}=M_{n, r, s}\left((\tau-y)^{2} ; y\right)$

$$
=\frac{1}{A_{r}(n y)}\left\{\frac{(2 s) !}{n^{2 s}} A_{r, 2 s}(n y)-y^{2 s}\right\}
$$

\section{Proof}

By using the same manner in theorem 3.4 and by applying Lemma 2.3 we get the proof.

\section{Example}


For $n=10,30,60$ the sequences $M_{n, r, s}\left(f_{1} ; y\right)$ converge to the function $f_{1}(\tau)=\sin (10 \tau), \tau \in[0,2]$ at $s=1,2$, and 3 , and $r=1,2$ in figures 1 , and 2 . The comparison between these sequences by the error functions $E(y)=\left|M_{n, r, s}\left(f_{1} ; y\right)-f_{1}(\tau)\right|$ in figures 3 and 4 .

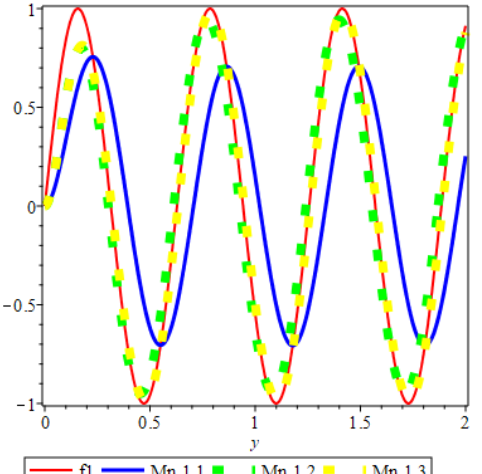

(a1)

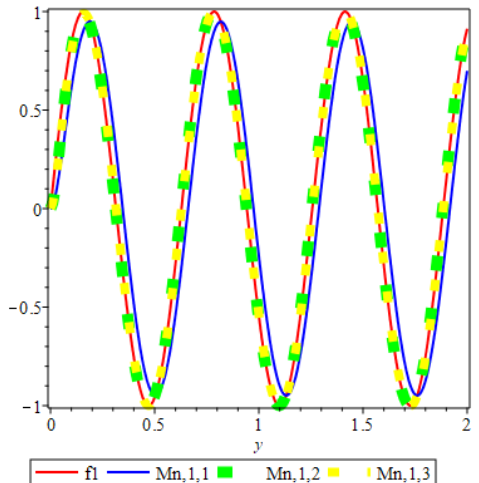

$(b 1)$

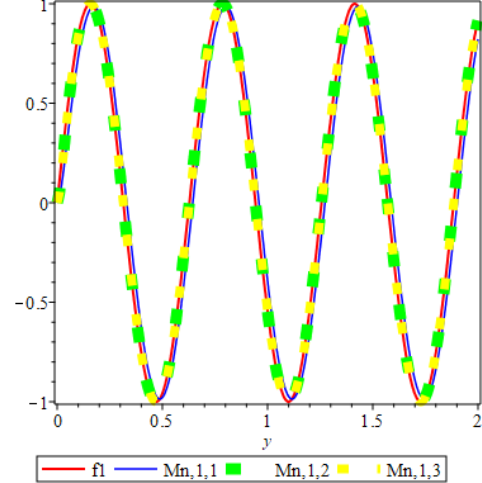

$(c 1)$

Fig. 1: The convergence of $M_{n, r, s}\left(f_{1}(\tau) ; y\right)$ at $r=1$ with $s=1,2$ and 3 at $n=10$ in $(a 1)$, at $n=30$ in $(b 1)$, and $n=60$ in (c1) respectively.
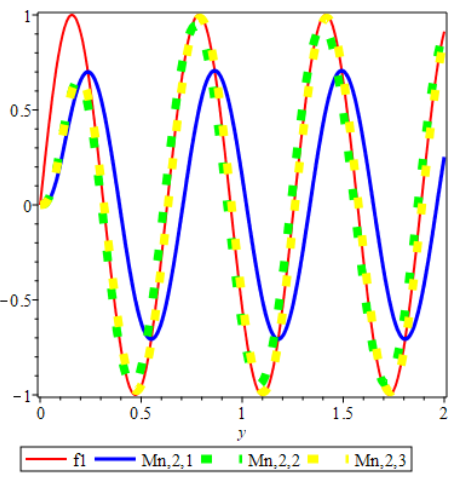

(a2)
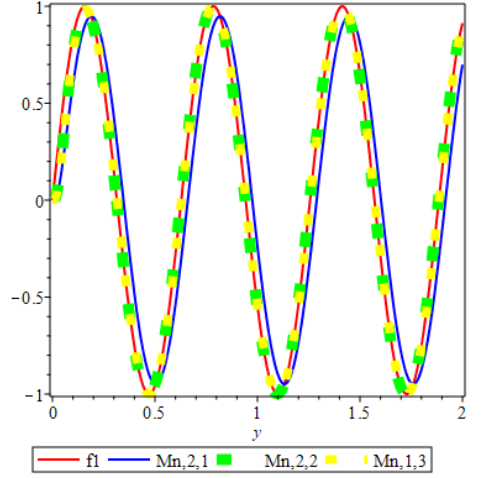

(b2)
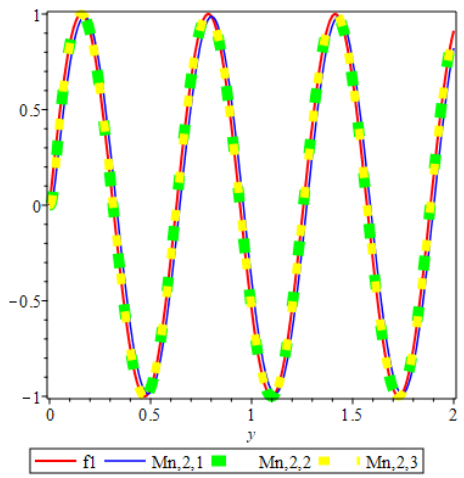

(c2)

Fig. 2: The convergence of $M_{n, r, s}\left(f_{7}(\tau) ; y\right)$ at $r=2$ with $s=1,2$ and 3 at $n=10$ in (a2), at $n=30$ in (b2), and $n=60$ in (c2) respectively.

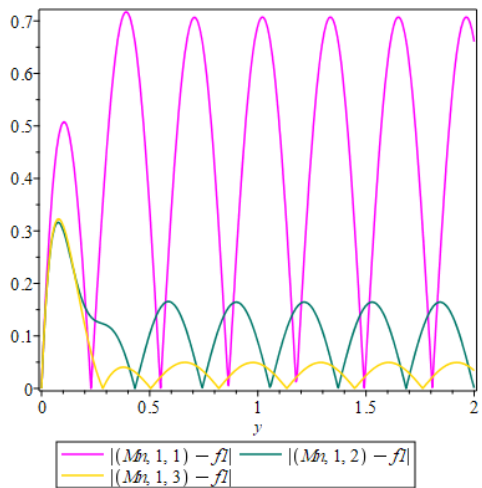

(a3)

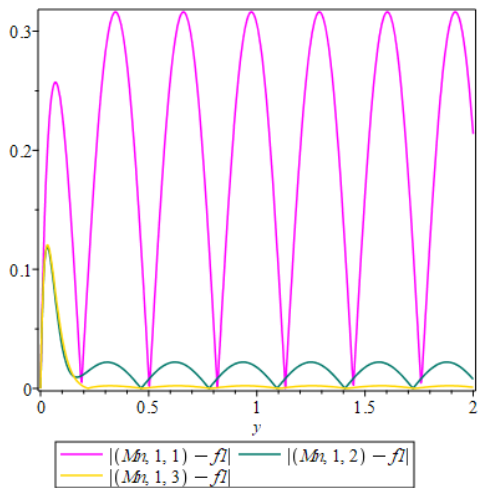

(b3)

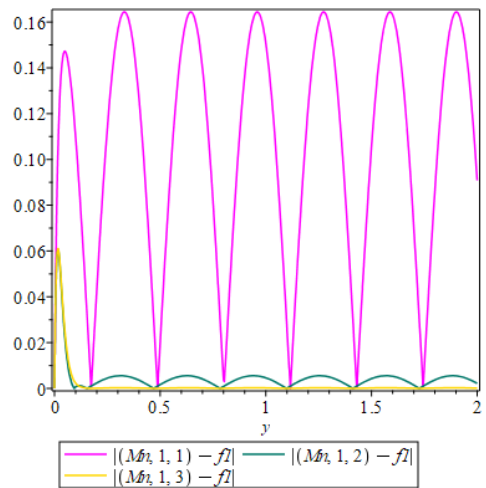

(c3)

Fig. 3: The error function $E(y)$ for the sequence $M_{n, r, s}\left(f_{7}(\tau) ; y\right)$ at $r=1$ with $s=1,2$ and 3 at $n=10$ in $(a 3)$, at $n=30$ in (b3), and $n=60$ in (c3) respectively. 


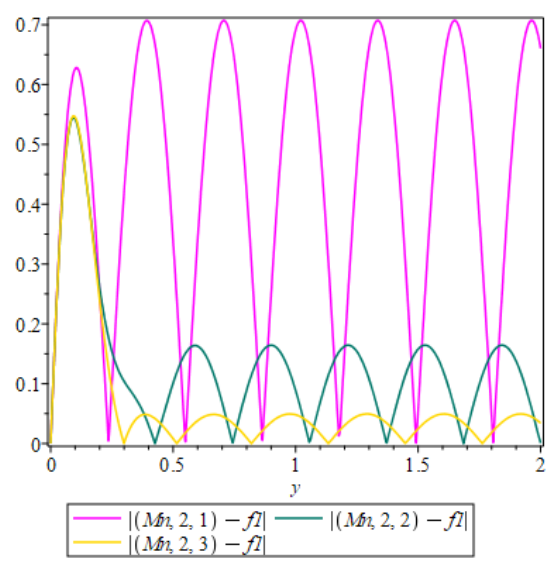

(a4)

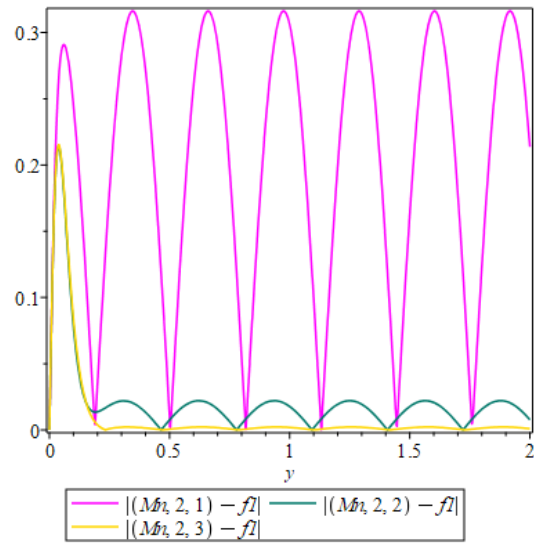

(b4)

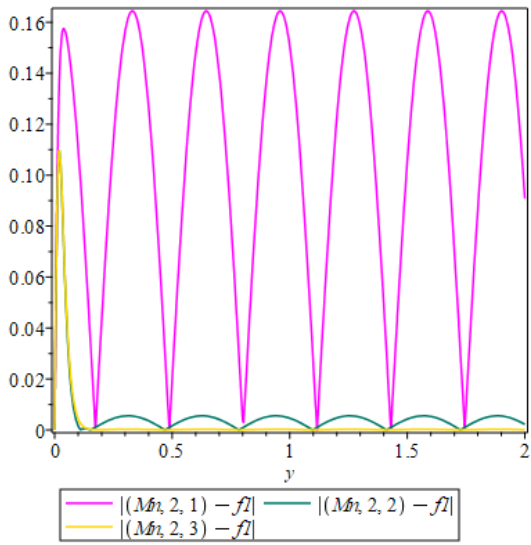

(c4)

Fig. 4: The error function $E(y)$ for the sequence $M_{n, r, s}\left(f_{7}(\tau) ; y\right)$ at $r=2$ with $s=1,2$ and 3 at $n=10$ in (a4), at $n=30$ in (b4), and $n=60$ in (c4) respectively.

\section{Conclusions}

We gave a numerical example for the sequence $M_{n, r, s}(f ; y)$ to approximate the test function $f(\tau)=\sin (10 \tau)$ in cases $n=10,30$ and 60 with arbitrary $r=1,2$ respectively for some values of $s=1,2,3$ and compared the results, it turns out the sequence $M_{n, r, s}(f(\tau) ; y)$ gave better results when $s$ is bigger.

\section{References}

1. S. S. Abed, Z. H. Maibed ed, Convergence Theorems of Iterative Schemes For Nonexpansive Mappings, Journal of Advances in Mathematics. Vol. 12, No. 12 (2010).

2. O. Duman and M. A. Ozarslan, Szasz-Mirakjan type operators providing a better error estimation, Applied Mathematics Letters 20(2007) 1184-1188.

3. V. Gupta, Rate of convergence for the modified Szasz-Mirakyan operators on of functions bounded variation, Journal of Mathematical An-Analysis and Application 233,476-483(1999).

4. H. S. Kansans Prasad, G. P. N Agrawal., and A. Sahai, On modified Szasz operators, (Proceedings of the International Conference on Mathematical Analysis and its Application) Kuwait Eds. S. M. Mazhar, A. Hamoui, and N. S. Faour, Pergamon, Press Oxford, 1985, pp. 29-41.

5. P. P Korovkin, Linear operators and Approximation Theory, Hindustan Publ. Corp. Delhi, 1960 (Translated from Russian Edition of 1959).

6. A. J. Mohammad and A. K Hassan, Simultaneous approximation by a new sequence of integral type Vol. 22 (2019), No. 8, pp. 1569-1576. DOI: 10.1080/09720502.2019.1706860

7. A. J. Mohammad, K. D. Abood, Approximation of General Form for a Sequence of Linear Positive Operator Based on Four Parameters, Journal of Advances in Mathematics, Volume: 14 Issue: 02 (2018). DOI: https://doi.org/10.24297/jam.v14i2.7573

8. B. P. Padhy, U. K. Misra, M. Misra and S. K. Nayak, Degree of approximation of Conjugate Series of a Fourier Series by $(E, r)(N, p, q)$ Means, Journal of Advances in Mathematics. Vol 9, No 2(2014).

9. L. Rempulska. and S. Graczyk, Approximation by modified Szãsz Mirakjan operators, J. Inequal. Pure and Appl. Math. 10(3) Art. 61 (2009), pp. 8.

10. O. Szãsz, Generalization of S. Bernstein's polynomials to the infinite interval, J. Res. Nat. Bur. Standard, 45(1950), pp.239-245.

11. K. B. Tarish, M. J. Mohammed, Best co-Approximation and best Similutanuos co- Approximation in Intuttionistic Fuzzy Normed Linear Space. Journal of Advaces in Mathematic. Vol. 11, No. 6(2020). 